\title{
Smart Home Wireless Power Control Design Based on Internet of Things
}

\author{
Xinyi Qi \\ Information Engineering School \\ of Communication University \\ of China, Beijing 100024 \\ e-mail: qixinyi818@sina.com
}

\author{
Mindan Bai \\ Information Engineering School \\ of Communication University \\ of China, Beijing 100024 \\ e-mail:mdyx@sohu.com
}

\begin{abstract}
In view of the current intelligence lives in complex structure, such as the high cost of weakness, use of switch power supply and single chip design a wireless intelligent household system. It will control instructions through wireless remote control by way of the wireless controller sent to the module, again by wireless module will dictate the analytical to microcontroller, by the single chip microcomputer control switch power to control relay on or off, so as to achieve the intelligent control household appliances the purpose of the power switch.This paper introduces a smart home wireless power remote control system design based on MCU. The remote controller sends control instructions to the controller of wireless receiving module via wireless way. Then the wireless receiving module parses the instructions and sends them to MCU. Take advantage of MCU to control relays of switch power on and off so as to control appliances switch intelligently. The feature of this system is low cost, simple design and can realize multi-channel control.
\end{abstract}

Keywords--power ; remote control ; MCU ; reception ; emission

\section{INTRODUCTION}

Along with the accelerated process of social informatization, the way people work and live has increasingly closer relationship with communication and information. Information society is not only changing people's lifestyle and work habits, but also challenging traditional residence. Social, technical and economic progress is also making huge changes on people's minds. Physical space is no longer the only requirement of residence, people pay more attention to safety, convenience and comfortable home environment. Smart home technology is designed by the concept of home.[1]

As a relatively new topic compared with the conventional remote control mode, remote control shows some advantages: less dependence on specialized wiring and less occupation of radio frequency resources .Thereby the remote control avoids electromagnetic pollution. At the same time, the existing telephone network can be fully used of due to telephone lines connecting to the internet all over.Thus, the remote control distance expands to the range of city and province, even across the countries. In addition, the telephone is duplex communication means. So it can obviously reflect the advantage of using a mobile phone to remote control. The operator can obtain a variety of instant information from controlled object through notification tone so as to take further operation. Using MCU to take an intelligent control, operator can take advantage of different notification tones to get information feedback according to different operating tips and the condition of the controlled object, which allow operator to keep abreast of the controlled object and make information controlled interactively and intelligently. [2][3]

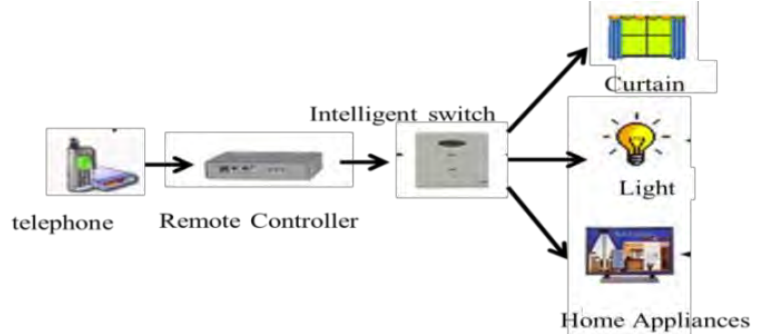

Figure 1. smart home control system diagram

consists of system server, family controller (various modules), various routers, cable modem head end equipment CMTS, switch, communicator, controller, wireless transceiver, various detectors, various sensors, various implementing agencies, printer and so on.[4]

\section{CONTROL SYSTEM TOTAL DESIGN}

The system consists of 3 parts: wireless transmitting module, wireless receiving module and switch power supply control module, the system structure is shown in Fig.2.

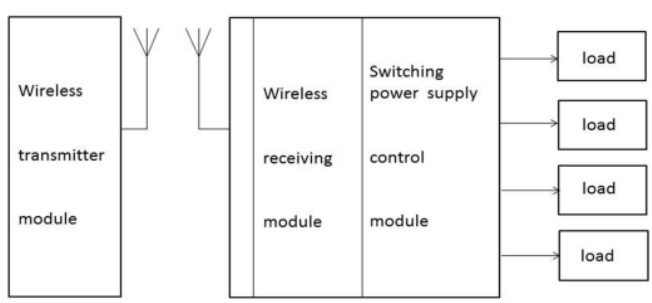

Figure 2. Control system structure

sending on and off instructions to a load 
circuit .Receiving module receives these instructions from transmitting module and then sends them to the processor of switch power supply control module. After parsing these instructions, processor controls relays of power supply module on and off, so as to remote control the load power intelligently.

\section{HARDWARE CIRCUIT DESIGN}

\section{A. Wireless transmitting module design}

Transmitting module is mainly composed of key circuit, coding circuit and wireless transmitting circuit. The main function of transmitting system is encoding the signal from key circuit to obtain encoded circuit pulse signal which will be modulated by wireless transmitting circuit and transmitted. Encoding circuit uses code chip PT2262 of the codec chipset PT2262 / 2272. Inside the chip there is oscillator, system timer, address decoder, encode pulse generator and control logic circuit. Power supply voltage of transmitting module is 5 volts, powered by a battery. Coding function depends on chip PT2262 to complete. The encoded signal sent by code chip PT2262 consists of address code, data code, synchronous code to be a complete code.

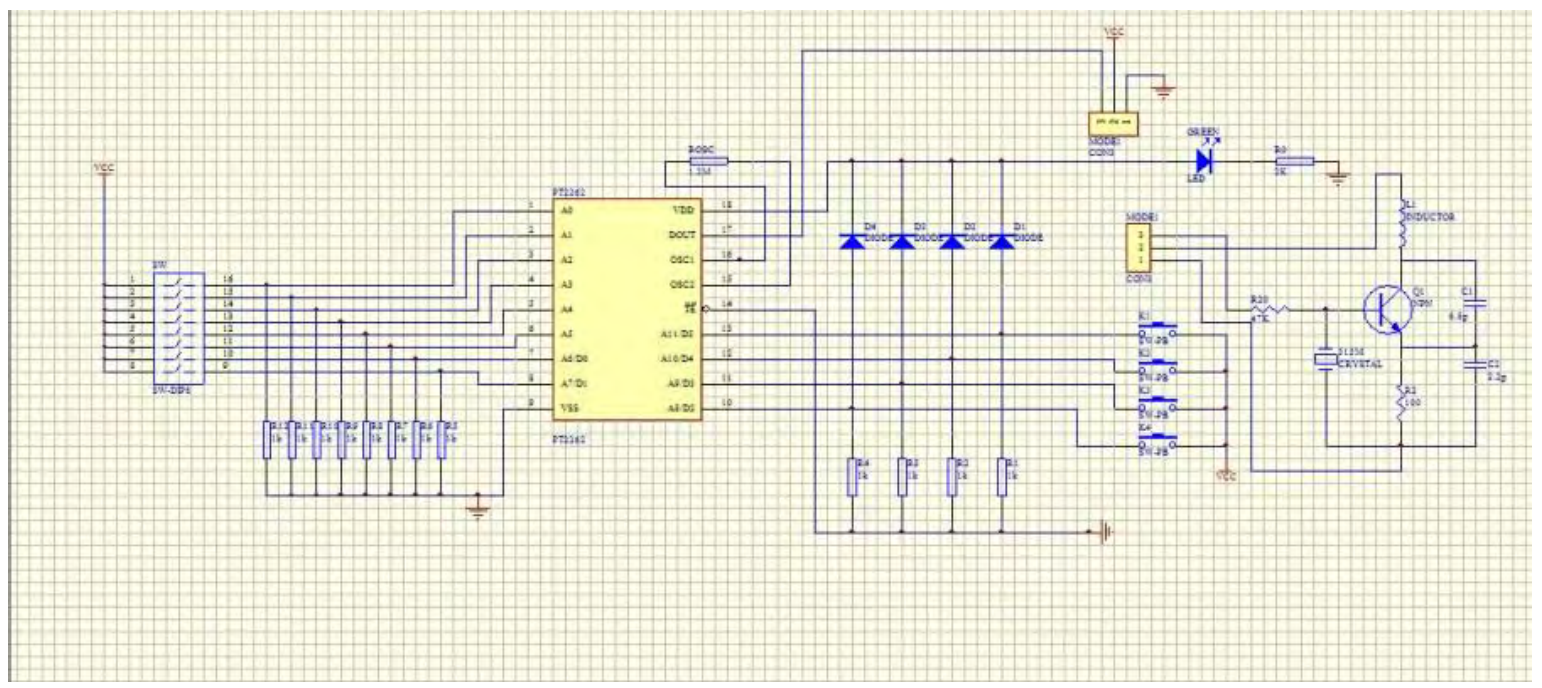

Figure 3. Transmitter module circuit

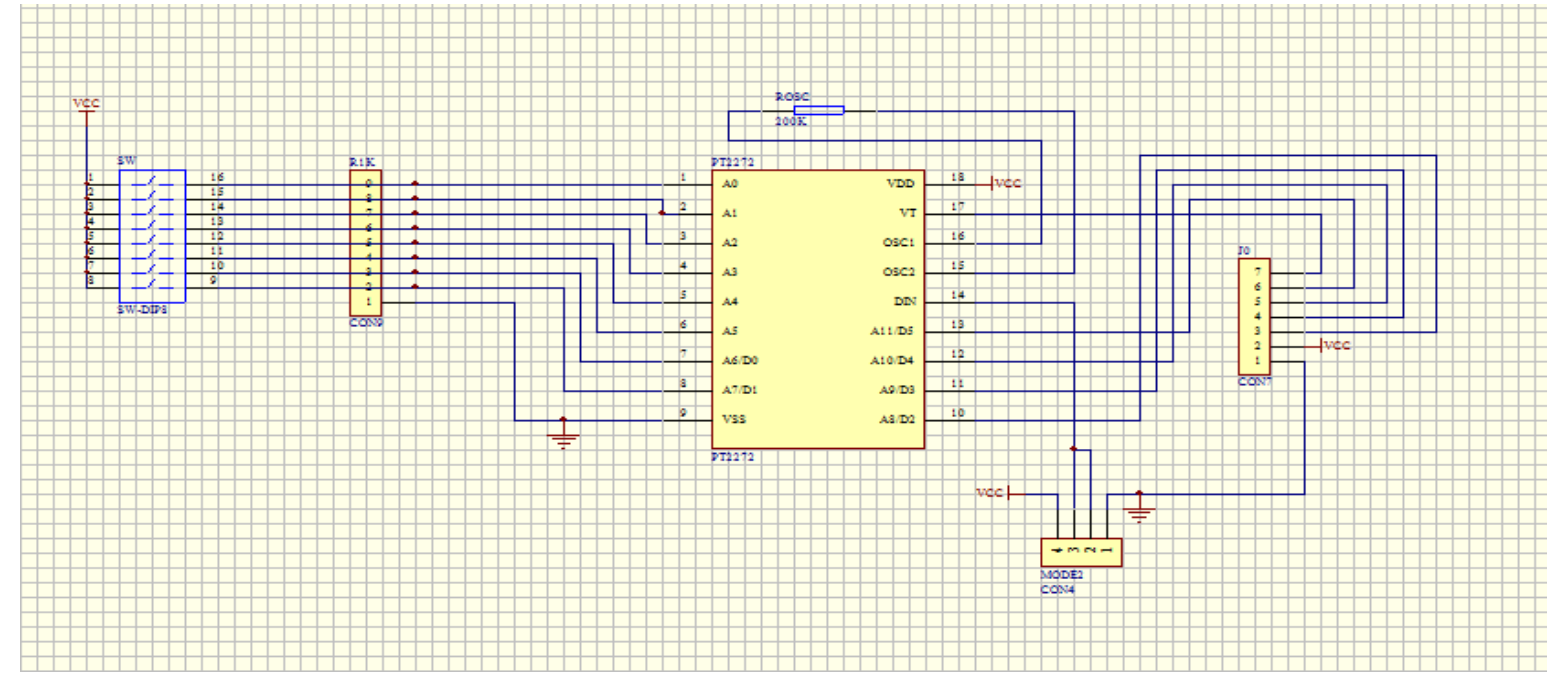

Figure 4. Receiving module circuit

Through the deep analysis of the structure of 2262 encoded $315 \mathrm{Mhz}$ data code segments, we can find that each of the code digit is fluctuated from low level to the high level, and then back to the low level, and then to the high level again. From the code pattern table, we can also find that each code digit can be divided into two sections. 
TABLE I. CODE DIGITS CORRESPONDING RELATION TABLE

\begin{tabular}{|c|c|c|c|c|}
\hline Code bits & $\begin{array}{c}\text { First } \\
\text { paragraph }\end{array}$ & Second & $\begin{array}{c}\text { Numerical } \\
\text { representation }\end{array}$ & $\begin{array}{c}\text { Anti- code } \\
\text { representation }\end{array}$ \\
\hline 0 & Narrow & Narrow & 00 & 11 \\
\hline 1 & Wide & Wide & 11 & 00 \\
\hline f & Narrow & Wide & 01 & 10 \\
\hline Invalid & Wide & Narrow & 10 & 01 \\
\hline
\end{tabular}

\section{B. Wireless receiving module design}

Receiving module is responsible for receiving signal from transmitting module, using chip PT2272 to decode the received signal. After checking the signal address code which has be set before whether matches with its own address code, PT2272 chip decodes the data. After that, the signal will be sent to processor to handle and then amplified by amplifier. Eventually, the processed signal controls relays on and off. The circuit can transform $220 \mathrm{~V}$ AC voltage into $5 \mathrm{~V}$ DC voltage and supply power to receiving module and processor of control module respectively.

\section{Control Module Design}

The switch power supply is mainly composed of MCU processor, relays and peripheral components, which are all from control module. This system can control multiple loads. Every time the control module connects to a relay indicating that a load can be controlled individually.

\section{Control Module Design}

The switch power supply is mainly composed of MCU processor, relays and peripheral components, which are all from control module. This system can control multiple loads. Every time the control module connects to a relay indicating that a load can be controlled individually.

As a core component, MCU is the main part of the control module. There are series of peripheral circuit design based on MCU. They are reset circuit, clock oscillating circuit. MCU P1 port is set as control signal input terminal, P0 port is set as display output terminal, $\mathrm{P} 2$ port is set as relays control signal output terminal. P2.0 outputs direction control signal, P2.1 outputs signal to control on / off.

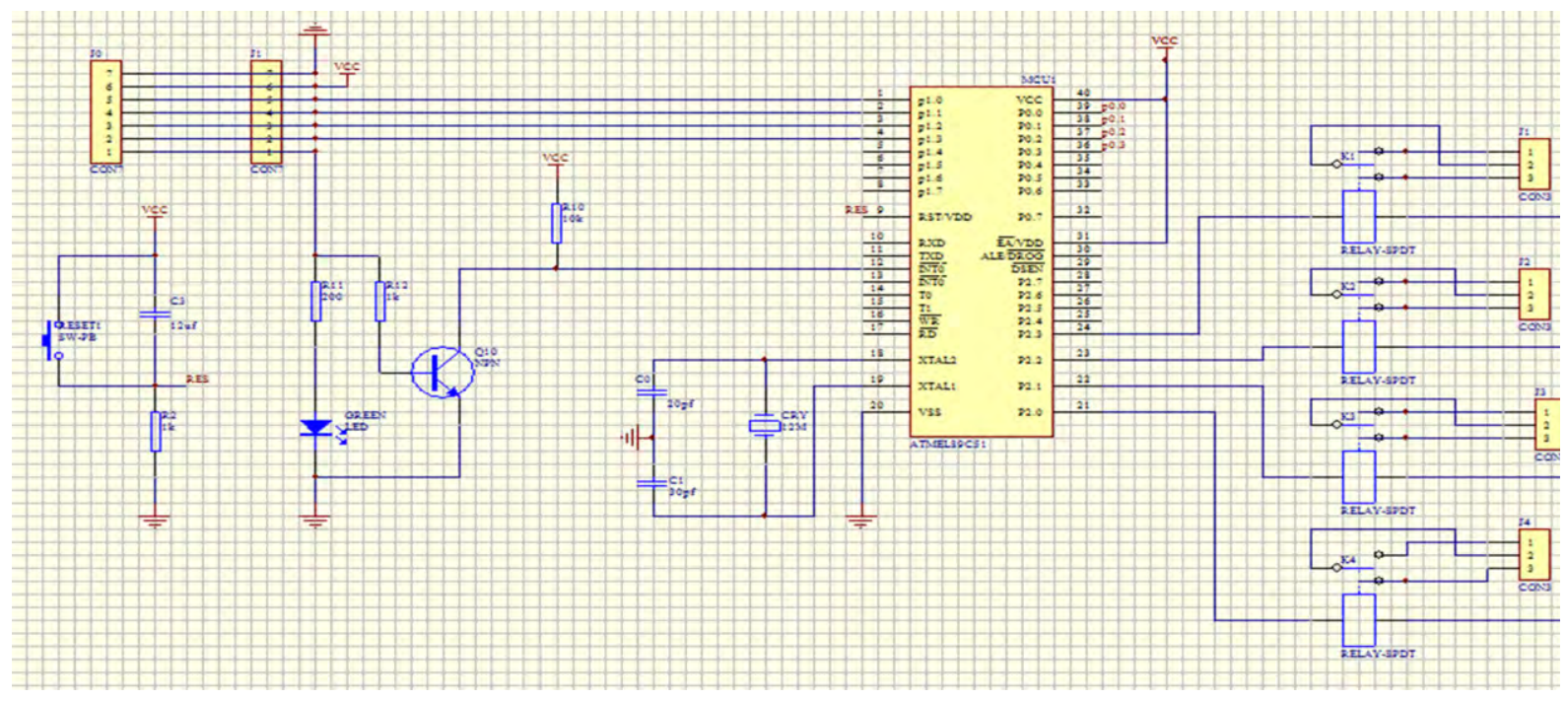

Figure 5. Control module circuit

\section{E. System Software Design}

Software design uses modular structure, adopts ST Visual Develop development environment as main workflow software and uses $\mathrm{C}$ language to do modular program, bringing great convenience during debugging the system. After the MCU power on, each module is initialized including clock initialization, port initialization and variable initialization. When the initialization is completed, the program enters the main loop, executing remote control decoding procedure and key judgment procedure. The program performs remote decoding procedures and key judgment programs successively when there is no button pressed or no remote data obtained; while an operation is taken, the program enters mode to judge the state. MCU saves remote control key data in the internal EEPROM to ensure the data won't lose if power supply lose. When a matched remote control key is pressed, the corresponding lamp will be on or off. After the operation is completed, program enters main loop again, executing remote decoding procedures and key judgment procedures. The flow chart is shown in Fig.6. 


\section{CONCLUSION}

The smart home control system was tested on the real circuit, a controller connected to four relays to control four $100 \mathrm{~W}$ lamps which are as loads to debug, four relays controlled four lamps respectively. The system used remote controller to control four lamps on and off, signal received well in the distance of $20 \mathrm{~m}$ in the empty

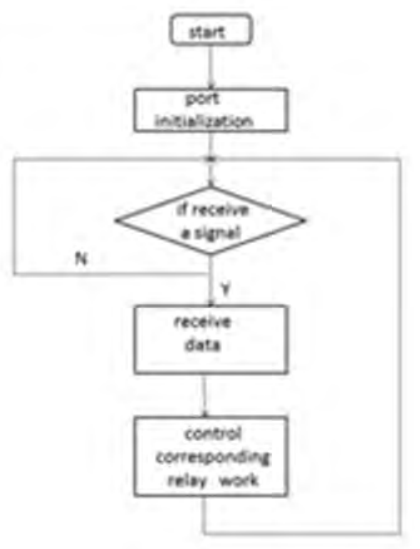

Figure 6. Software design flow

room. The experiment achieved the desired effect, providing the foundation for next study. Smart home wireless remote control technology is still under continuous development and improvement at present, encountering some problems in the practical application. For example, some higher-level function applications such as high-quality image transmission are still difficult to achieve because of the interference from current wireless network signal and constraints of transmitting bandwidth and stability. But with the communication network completing gradually in our country, all these constraints will be solved, smart home and wireless remote control technology will get a bigger development.

\section{REFERENCE}

[1] DALAS Semiconductor.Atomatic Identification Data Book[M] .2009.

[2] BUSHBY S T. A standard communication infrastructure for intelligent Buildings $[\mathrm{J}]$. Automation in Construction, 2011, 6 : $529-540$.

[3] GUREN G, NOZAL L A L, BLAZQUEZ A. Remote control of sensors and actuators by GSM[J]. Industrial Electronics Society, 2002, $11: 2306-2310$

[4] CHOY C S. An infra-red remote control system designed for universal control $[\mathrm{J}]$. Consumer Electronics, 2010, 11 : $1089-1094$.

[5] Socheat Heng, Cong-Kha Pham. Improvement of LDOS PSRR Deteriorated By Reducing Power Consumption : Implementation and Experimental Results. IC Design and Technology, 2009. ICICDT'09. IEEE International Conference. 2009 : $11 \sim 15$

[6] Huei-Sheng Jhuang, Jia-Hui Wang, Zi-Yu Zeng etc. A low dropout linear regulator with hi power supply rejection. Integrated Circuits, ISIC'09. Proceedings ofthe 2009 12th International Symposium. 2009: 41 44

[7] Chenchang Zhan, Wing-Hung Ki. A low dropout regulator for SoC with power supply rejection and low quiescent current. Integrated Circuits, ISIC'09. Proceedings ofthe 2009 1 2th International Symposium. $2009: 37 \sim 40$

[8] Mohammed Usaid Abbasi, Tanvir Ahmad Abbasi, Mohammed Suhaib Abbasi etc. An Improved Fast Transient Response Low Dropout Regulator. Circuits and Systems, 2009. ISCAS 2009. IEEE International Symposium. 2009 : 808-8 11

[9] Dae-Man Han , Jae-Hyun Lim . Smart home energy management system using IEEE 802. 15.4 and zigbee[C]. Consumer Electronics, 2010, v01 . 56(3) : $1403 \cdot 1410$.

[10] Popa, M, Popa, A. S, Marcu, M. A distributed smart card based access control systemic Intelligent Systems and lnformatics, 2010 , v01. 8, 34 1-346.

[11] 30.Siemens AT Command Set Siemens Cellular Engines TC35 module,TC37 module and TC35 Terminal version:03.10,DocID:TC3X ATC 01V03.10

[12] XIA Tao, TAO Yang. Based on four modules of the intelligence lives in research and design[J1 . Engineering Techonology, 2011.25(3) : 52-55. 